ARTICLE

https://doi.org/10.1038/s41467-019-12297-0

\title{
Non-enzymatic roles of human RAD51 at stalled replication forks
}

\author{
Jennifer M. Mason ${ }^{1,2,3}$, Yuen-Ling Chan', Ralph W. Weichselbaum¹ \& Douglas K. Bishop ${ }^{1,4}$
}

The central recombination enzyme RAD51 has been implicated in replication fork processing and restart in response to replication stress. Here, we use a separation-of-function allele of RAD51 that retains DNA binding, but not D-loop activity, to reveal mechanistic aspects of RAD51's roles in the response to replication stress. Here, we find that cells lacking RAD51's enzymatic activity protect replication forks from MRE11-dependent degradation, as expected from previous studies. Unexpectedly, we find that RAD51's strand exchange activity is not required to convert stalled forks to a form that can be degraded by DNA2. Such conversion was shown previously to require replication fork regression, supporting a model in which fork regression depends on a non-enzymatic function of RAD51. We also show RAD51 promotes replication restart by both strand exchange-dependent and strand exchange-independent mechanisms.

\footnotetext{
${ }^{1}$ Department of Radiation and Cellular Oncology, University of Chicago, Chicago, IL, USA. ${ }^{2}$ Department of Genetics and Biochemistry, Clemson University, Clemson, SC, USA. ${ }^{3}$ Center for Human Genetics, Clemson University, Clemson, SC, USA. ${ }^{4}$ Department of Molecular Genetics and Cell Biology, University of Chicago, Chicago, IL, USA. Correspondence and requests for materials should be addressed to J.M.M. (email: jmason4@clemson.edu) or to D.K.B. (email: dbishop@uchicago.edu)
} 
$\mathrm{T}$ he complete and accurate replication of the genome is essential to maintain genome integrity. Replication forks face many obstacles that can result in replication fork stalling or replication fork collapse. Proteins initially identified on the basis of their roles in homologous recombination (HR) are now known to have key functions during replication stress ${ }^{1,2}$. HR proteins act to protect and remodel stalled replication forks, and reconstruct functional replication forks following fork collapse. As a result of these activities, HR proteins are critical to the ability of cells to restart stalled and collapsed replication forks.

The central HR protein, RAD51, forms helical nucleoprotein filaments on tracts of single-strand DNA (ssDNA), such as those formed by nucleolytic processing of the DNA ends at the sites of DNA double strand breaks (DSBs). Once RAD51 filaments form on tracts of ssDNA, the protein alters the structure of the ssDNA, allowing the nucleoprotein filament to catalyze a homology search to identify an identical or nearly identical sequence in duplex DNA, and then carry out exchange of the bound ssDNA strand with the like strand of the homologous duplex ${ }^{3}$. In this way, the homology search and strand exchange activity of RAD51 acts to form a homologous joint between a broken chromatid and its intact sister chromatid, leading to accurate repair of the DSB.

In addition to its role in repair of DSBs, RAD51 has three separate roles at stalled replication forks. First, RAD51 promotes replication fork regression, a process that has also been referred to as replication fork reversal ${ }^{4,5}$. Replication fork regression involves branch migration in the direction opposite to replication to form a Holliday junction-containing chicken foot structure. Second, RAD51 protects tracts of newly synthesized DNA from degradation. Initial resection of the reversed forks by MRE11, EXO1, and DNA2 produces a ssDNA overhang at regressed forks 5 . The formation of a stable RAD51 filament on the resulting ssDNA overhang prevents extensive degradation of regressed forks. In a subset of tumor cell lines, nascent ssDNA degradation occurs in cells with partial inhibition of RAD51 expression or activity in response a variety of DNA damage agents ${ }^{7-12}$. Nascent DNA degradation also occurs in cells lacking proteins required to load RAD51 on ssDNA, such as BRCA1, BRCA2, FANCD2, and the RAD51 mediators including RAD51C and XRCC25,7,12-16. The degradation phenotype observed in these cells results from the inefficient nucleation and/or stabilization of RAD51 filaments at the regressed fork ${ }^{5,15-17}$. Three nucleases involved in DNA end resection, MRE11, EXO1, and DNA2 are responsible for the degradation of nascent DNA strands. MRE11 and EXO1 degrade forks in cells with defects in RAD51 filament formation and stabilization. DNA2 degradation has been observed under two conditions. First, mutant cells in which RAD51 is efficiently loaded onto reversed forks, but filament stability is decreased results in DNA2-dependent degradation ${ }^{18}$. Second, in U20S cells, prolonged HU treatment results in DNA2-dependent degradation that occurs even without manipulations that decrease RAD51 filament stability ${ }^{6,11}$. Depletion of RADX, a single-stranded binding protein that negatively regulates RAD51 at regressed replication forks, prevents excessive MRE11- and DNA2-dependent degradation that results from defects that reduce RAD51 filament formation or RAD51 filament stability ${ }^{11}$. However, depletion of RADX did not prevent DNA2-dependent degradation after prolonged $\mathrm{HU}$ treatment, indicating degradation occurs independently of RAD51 filament stability. Finally, RAD51 plays a role in restart of stalled or collapsed replication forks ${ }^{19}$. Collapse of replication forks may sometimes occur as a consequence of collision of forks with a single-strand nick in the template and has been to shown to occur as a consequence of enzymatic cleavage of reversed forks by structure-specific endonucleases including MUS81 ${ }^{20-22}$. Repair of the resulting DSB by HR to reinstate the replication fork requires RAD51 and MRE11 activity $19,23,24$.
Although it is clear that RAD51 is required for protection of nascent DNA strands from MRE11, replication fork remodeling, and fork restart, the molecular mechanisms underlying RAD51's role in each of these functions remains to be determined. One particularly important question is whether and to what degree RAD51's homology search and strand exchange activity, i.e., it is enzymatic activity, is required for each its roles during the replication stress response. The ability of RAD51 to promote nuclease protection at stalled forks and/or HR can be separated from its ability to promote fork regression. Partial knockdown of RAD51 resulted in MRE11-dependent degradation of replication forks, but knockdown to low levels of RAD51 rescues fork deprotection by preventing replication fork regression ${ }^{11}$. RAD51dependent replication fork regression is BRCA2-independent ${ }^{5,17}$ even though protection of regressed forks from pathological degradation by MRE11 is BRCA2-dependent. In addition, replication-associated sister chromatid exchange has been observed in BRCA2-deficient cells that display defects in DSBdependent HR and RAD51 immunostaining focus formation ${ }^{25}$. Another important study showed cells that co-express RAD51 WT with RAD51 T131P, a dominant-negative allele of RAD51 that forms unstable nucleoprotein filaments due to constitutive ATPase activity, are HR proficient, undergo fork regression, but do not protect forks from degradation by MRE11 or DNA2 $2^{5,11,18}$. However, the ability of RAD51 T131P to specifically disrupt fork protection is only seen in cells co-expressing RAD51 WT at a ratio that delays RAD51 focus formation, but does not inhibit HR (e.g., in heterozygous, patient-derived cells) ${ }^{18}$; RAD51 T131P protein does not form immunostaining foci and high levels of mutant protein inhibits focus formation of endogenous RAD51. This finding suggests that RAD51 T131P partially inhibits the function of wildtype RAD51 resulting in the observed separation of function. Another mutant allele of RAD51, RAD51 K133R, which is defective in ATP hydrolysis, was found to rescue MRE11-dependent fork protection in FANCD2-deficient and BRCA2-deficient cells. However, human RAD51 K133R retains strand exchange activity in vitro ${ }^{26-28}$ and can promote significant amounts of HR in vivo, although human RAD51 K133Rmediated HR has only been observed in chicken DT40 cells ${ }^{27}$. Given that RAD51 K133R lacks ATPase activity which is involved in promoting filament disassembly ${ }^{26}$, it is possible that the HR defect observed in human cells expressing the mutant protein results from a post strand exchange block to RAD51 filament disassembly rather than from a defect in strand exchange. Together, these considerations led us to the conclusion that the prior work which separated different functions of RAD51 during replication stress did not specifically test the role of the protein's enzymatic homology search and strand exchange activity in any of the protein's replication-associated functions. In this context it should be noted that both ensemble and single molecule biochemistry have shown that only $8 \mathrm{bp}$ of homology are required for meta-stable homology-recognition by the enzymatic activity of RAD51 family strand exchange protein ${ }^{29,30}$. Given the binding stoichiometry of RAD51 to DNA (1 protomer per 3 nucleotides), filaments too short to be detected as immunostaining foci could, in principle, promote strand exchange at stalled replication forks. Thus, we propose that strand exchange could be a residual activity of partially defective RAD51 during replication stress, even in cells defective in RAD51 focus formation, such as BRCA2-deficient cells.

During the strand exchange reaction, RAD51 utilizes two distinct binding sites. RAD51 forms nucleoprotein filaments on resected single-strand DNA via a high-affinity binding site (site I). The RAD51 nucleoprotein filament searches intact dsDNA for homologous sequences by binding a second low affinity binding site (site II), consisting of a cluster of positively charged residues 
that lie on the interior surface of the helical filament ${ }^{31}$. This cluster binds backbone phosphates of dsDNA regions being searched, and also continues to bind the out-going ssDNA strand once strand exchange occurs ${ }^{32}$. In Saccharomyces cerevisiae, RAD51 containing mutations in site II (II3A) retains the ability to form nucleoprotein filaments, but is fully defective in homology search and strand exchange in vitro and highly defective in mitotic HR in vivo ${ }^{31}$.

Here, we disrupt the secondary binding site on human RAD51 to determine the role of strand exchange activity in response to replication stress, focusing on the well-studied human osteosarcoma epithelial cell line, U2OS. Our results provide definitive evidence that the enzymatic activity of RAD51 is not required to protect stalled forks from MRE11 degradation, as expected. Surprisingly, we also show the enzymatic activity is not required for RAD51-dependent remodeling of stalled forks to a form that permits DNA2-dependent degradation of nascent DNA. In contrast, we provide evidence that RAD51 promotes replication restart by both strand exchange-dependent and strand exchangeindependent mechanisms. Cytological and knockdown experiments show that cells expressing a strand exchange-defective form of RAD51 accumulate collapsed replication forks and undergo frequent new origin firing in response to $\mathrm{HU}$-induced replication stress.

\section{Results}

hsRAD51-II3A binds DNA, but is defective in D-loop formation. To characterize the molecular functions of human RAD51's DNA binding and strand exchange activities during replication stress, we constructed an allele of human RAD51 corresponding to the S. cerevisiae rad51-II3A allele ${ }^{31}$. The corresponding human RAD51-II3A (hsRAD51-II3A) protein has three amino acid residues, R130, R303, and K313 changed to alanines. To determine if the mutant human protein (hsRAD51-II3A) had the same properties as its budding yeast counterpart, we purified hsRAD51-WT and hsRAD51-II3A proteins (Supplementary Fig. 1a). We analyzed the nucleoprotein filament forming and strand exchange activities of the two forms of RAD51 using biochemical assays. Using fluorescence polarization (FP), we measured binding of the two forms of the protein to a Alexa84tagged 84-nt ssDNA oligo ${ }^{33}$. Titration showed that the two proteins have similar binding activities to this oligo, the apparent $K_{\mathrm{d}}$ 's for hsRAD51-WT and hsRAD51-II3A were $57 \pm 1 \mathrm{nM}$ and $132 \pm 5 \mathrm{nM}$, respectively (Fig. 1a). Thus, hsRAD51-II3A displays only a modest DNA binding defect in this assay. Next, we examined the homology search and strand exchange activity of hsRAD51-II3A with a D-loop assay that employs a 90-nt singlestrand oligonucleotide and a 4.4-kb supercoiled plasmid carrying a dsDNA sequence identical to the sequence of the oligonucleotide (Fig. 1b). In this assay, hsRAD51-II3A exhibited 840 -fold less D-loop activity compared to hsRAD51-WT $(0.06 \%$ vs. $17 \%$ of plasmid DNA forming D-loops, respectively). Together the results demonstrate that, like its budding yeast counterpart, hsRAD51-II3A retains DNA binding, but not strand exchange activity in vitro.

Next, we asked if hsRAD51-II3A displays separation of RAD51's DNA binding and HR functions in vivo. hsRAD51WT and hsRAD51-II3A were expressed in U2OS cells and expression of the endogenous RAD51 protein was repressed via siRNA targeting of the $3^{\prime} \mathrm{UTR}$ of the RAD51 mRNA to less than $7 \%$ of that observed in the nonsilencing siRNA (siNS) control (Supplementary Fig. 1b). Both hsRAD51-WT and hsRAD51-II3A were expressed at the same level, which was approximately fivefold higher than that seen for the endogenous protein in the siNS control (Supplementary Fig. 1b).
To determine the extent to which the spontaneous distribution of RAD51 differed in cells transfected with RAD51 expression constructs from that observed with endogenous RAD51, we counted RAD51 foci in unselected nuclei. RAD51 typically forms a small number of nuclear immunostaining foci in the absence of induced damage at sites of spontaneous DNA damage or the sites of nonrepair-associated RAD51 oligomers. The number of spontaneous RAD51 foci between cells slightly increased in cells expressing hsRAD51-WT $(1.5 \pm 6.7$ (mean \pm STD) RAD51 foci/ cell) or hsRAD51-II3A (1.5 \pm 4 RAD51 foci/cell), when compared to siNS $(0.7 \pm 1.8 \mathrm{foci} / \mathrm{cell}$ in siNS cells; Fig. 1c, d). In addition, a small subpopulation of hsRAD51-WT $(3.4 \pm 1.5 \%)$ and hsRAD51-II3A $(2.3 \pm 1.3 \%)$ transfected cells contained an average of $44 \pm 14$ elongated RAD51 fibers with contour lengths of $0.5-3$ microns long (Supplementary Fig. 1c). This type of staining pattern was observed previously as a consequence of RAD51 overexpression and reflects binding of RAD51 to undamaged $\mathrm{DNA}^{34}$. This analysis indicated that the level of expression of RAD51 from transfection of siRNA resistant constructs causes only a slight increase in the frequency of spontaneous foci.

Analysis of damage induced RAD51 foci provided evidence that hsRAD51-II3A retains DNA binding activity in vivo. Loading of RAD51 protein filaments on the $3^{\prime}$ ssDNA overhang at resected DSBs in vivo is conventionally assayed by measuring the number of RAD51 foci resulting from treatment of cells with agents that induce DNA breaks, such as X-rays ${ }^{34-37}$. The number of RAD51 foci significantly increased after X-ray treatment of siNS transfected cells (Fig. 1c, d; $11 \pm 14.4$ foci/cell IR vs. $0.7 \pm 1.8$ foci/cell). Cells depleted of RAD51 exhibited a fourfold reduction in IR-induced RAD51 focus formation ( $2.5 \pm 6$ foci/cell; $p<0.005$; Mann-Whitney test). Cells transfected with hsRAD51-WT $(9 \pm$ 9.7 foci/cell) showed the same level of X-ray induced foci as the siNS control (11 \pm 14.4 foci/cell). Importantly, the number of IRinduced hsRAD51-II3A $(9 \pm 8.3)$ foci did not differ significantly from siNS or hsRAD51-WT controls. Together, these results indicate hsRAD51-II3A retains significant DNA binding activity in vivo.

To determine if hsRAD51-II3A is defective in HR, we employed the DR-GFP assay ${ }^{38}$. In this assay, HR generates a functional green fluorescent protein (GFP) allele following induction of a chromosomal DNA break in one of the two defective copies of GFP carried by the reporter cell line (Supplementary Fig. 1d). DSBs were induced in the U2OS-DR-GFP cells by transfection with a plasmid expressing the I-SceI endonuclease. HR efficiency was then measured by flow cytometry as the frequency of GFPexpressing cells. RAD51 depletion reduced HR efficiency 6-fold compared to siNS controls $(0.97 \pm 0.1 \%$ GFP-positive cells siNS vs. $0.16 \pm 0.03 \%$ siRAD $51 ; p$ value $<0.05$; $t$ test; Fig. 1e). Expression of hsRAD51-WT in RAD51-depleted cells increased the HR efficiency by threefold compared to siRAD51 cells $(0.49 \pm 0.03$ GFP-positive cells vs. $0.16 \pm 0.03 \%$ siRAD51; $p$ value $<0.005$ ). In contrast, hsRAD51-II3A did not increase HR efficiency in RAD51-depleted cells $(0.12 \pm 0.09 \%$ GFP positive cells vs. $0.16 \pm 0.03 \%$; $p$ value $=0.6$ ) indicating hsRAD51-II3A is defective for HR-mediated repair of DSBs. Consistent with our biochemical observations, these data indicate human hsRAD51-II3A is able to form RAD51 nucleoprotein filaments with normal efficiency, but is defective for HR in vivo.

hsRAD51-II3A protects forks from degradation by MRE11. We next sought to elucidate the molecular function of RAD51's enzymatic activity during perturbed replication, using the DNA fiber assay to measure the ability of cells treated with the replication inhibitor HU to protect nascent DNA strands from degradation. Nascent DNA undergoes MRE11-mediated 
a

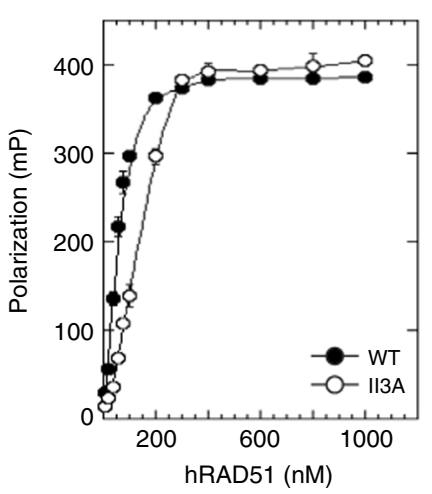

C

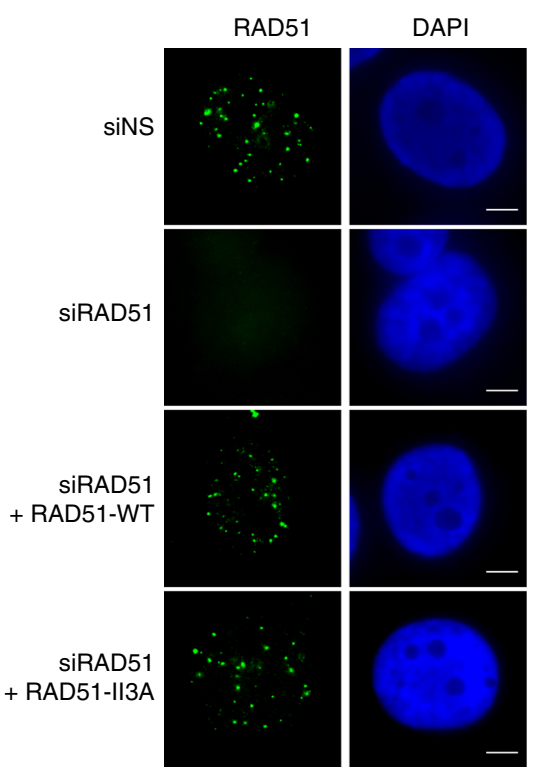

6 Gy X-rays b
hRAD51 $(\mu \mathrm{M}) 0 \frac{\mathrm{WT}}{0.511 .52} \frac{\| 3 \mathrm{~A}}{0.511 .52}$

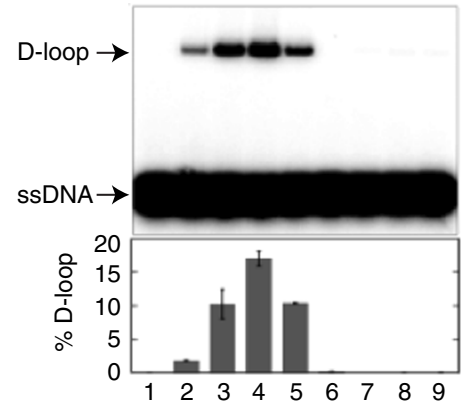

d
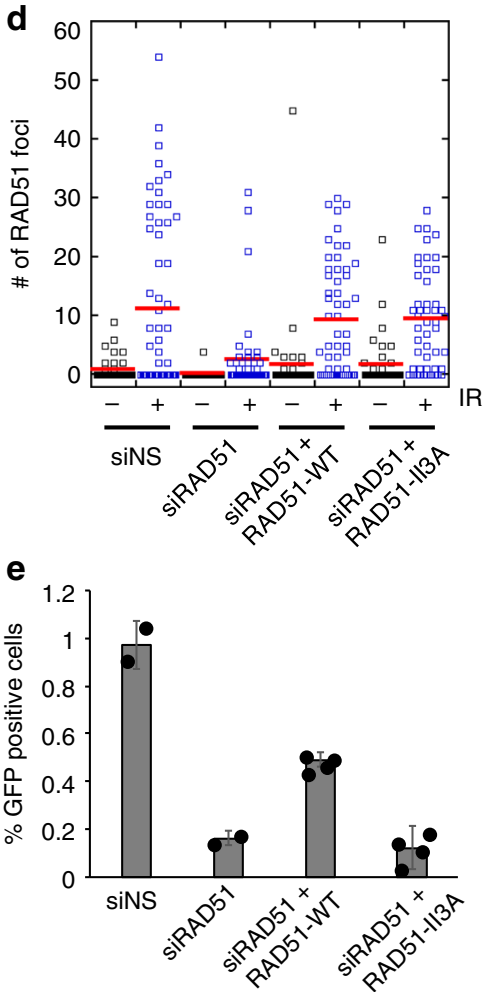

Fig. 1 hsRAD51-II3A retains ssDNA binding activity, but is defective for HR. a Human RAD51 binding to ssDNA was determined by fluorescence polarization. Protein at various concentrations was incubated with fluorescein-tagged 84-mer ssDNA (200 nM nucleotides or $2.4 \mathrm{nM}$ molecules) in buffer $\mathrm{B}$ and incubated at $37^{\circ} \mathrm{C}$ for $30 \mathrm{~min}$. Error bars represent standard error of the mean from triplicate experiments. The apparent $K_{d}$ for hsRAD51-WT is $57 \pm$ $1 \mathrm{nM}$, for hsRAD51-II3A is $132 \pm 5 \mathrm{nM}$. b The D-loop activity of hsRAD51-WT and hsRAD51-II3A was measured in the presence of increasing concentrations of protein. Upper panel: autoradiogram following electrophoretic separation of D-loops from free 32P-labeled ssDNA oligo substrate. Lower panel: quantitation of the autoradiogram shown in the upper panel. Error bars represent standard error of the mean from triplicate experiments. $\mathbf{c}$ Images depict immunostaining RAD51 in cells prepared for staining $8 \mathrm{~h}$ after a dose of $6 \mathrm{GY}$ X-rays in the indicated samples. Green-RAD51 Blue-DNA. Scale bar $=5 \mu \mathrm{m}$. d Quantitation of RAD51 foci after the indicated treatments. Red line represents the mean. $N=50$ nuclei from two independent experiments. Values are presented as mean \pm STD. Statistical significance was determined using Mann-Whitney test (e) DR-GFP assay. The percentage of GFP-positive cells in each sample are graphed. Graph is the average from two independent experiments. hsRAD51-WT and hsRAD51-II3A samples were done in duplicate for both experiments. Error bars represent standard deviation and values are presented as mean \pm SD. Statistical significance was determined using $t$ test.

Source data are provided as a source data file

degradation in cells that have defects in RAD51 loading and/or stabilization of RAD51 nucleoprotein filaments $5,7,13,15,16$. MRE11-dependent fork degradation in FANCD2 and BRCA2deficient cells can be rescued by overexpressing RAD517,13. We determined if hsRAD51-II3A overexpression rescues fork degradation in U2OS cells depleted of FANCD2 after treatment with 4 $\mathrm{mM} \mathrm{HU}$ for $5 \mathrm{~h}$ (Fig. 2a, Supplementary Fig. 2a). Consistent with previous studies, we observed replication tract shortening in FANCD2-depleted cells $(0.55 \pm 0.15 \mu \mathrm{m}$ (mean \pm STD) vs. $0.74 \pm$ 0.16 , $p$ value $<0.0001$, Mann-Whitney test). As expected, treatment with mirin $(0.78 \pm 0.15 \mu \mathrm{m}, p<0.0001)$ and expression of hsRAD51-WT $(0.79 \pm 0.18 \mu \mathrm{m}, p<0.001)$ restored replication tracts to lengths observed without $\mathrm{HU}$ treatment ${ }^{13}$. Expression of hsRAD51-II3A also restored replication tract length in FANCD2depleted cells. We verified this result by expressing hsRAD51-WT and hsRAD51-II3A in a BRCA2-deficient (VU423) fibroblast cell line, which has also been shown to undergo MRE11-dependent degradation upon HU-induced stress ${ }^{39}$ (Fig. 2b). As expected, HU treatment resulted in shortened replication tracts $(0.84 \pm$ $18 \mu \mathrm{m}$ vs. $0.62 \pm 23 \mu \mathrm{m}, p$ value $<0.0001)$ that were rescued by 
a
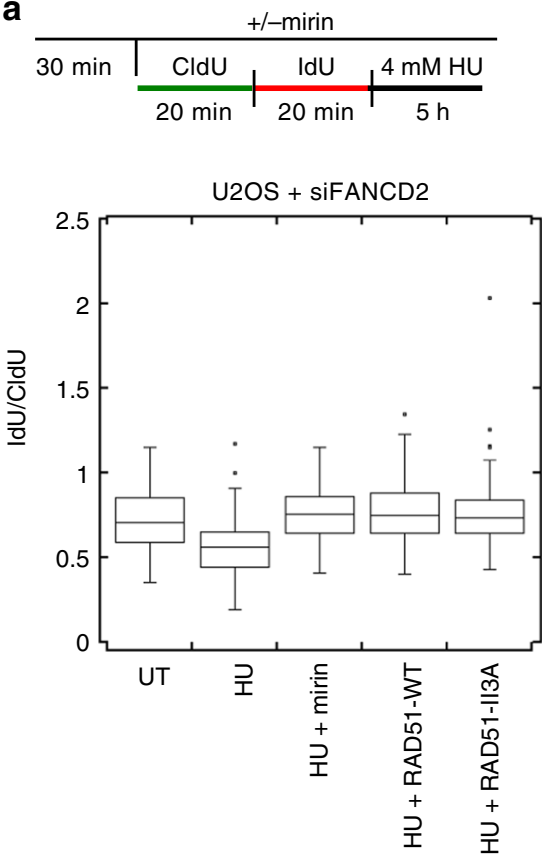

b

\begin{tabular}{|c|c|c|c|}
\hline \multicolumn{4}{|c|}{$+/-$ mirin } \\
\hline $30 \mathrm{~min}$ & CldU & IdU & $4 \mathrm{mM} \mathrm{HU}$ \\
\hline & & $\min$ & $5 \mathrm{~h}$ \\
\hline
\end{tabular}

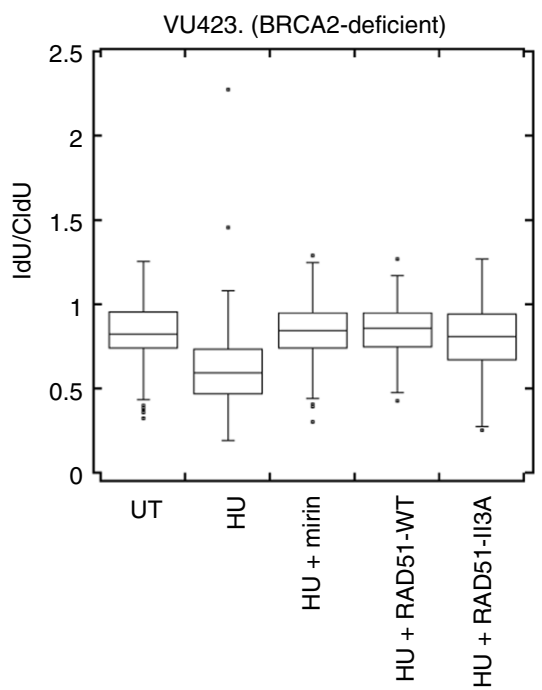

Fig. 2 hsRAD51-II3A protects nascent strands from MRE11-dependent degradation. a Box plot represents the IdU/CldU ratio of tracts measured in FANCD2depleted U2OS cells after the indicated treatment. b Box plot represents the IdU/CldU ratio of tracts measured in BRCA2-deficient fibroblasts (VU423) after the indicated treatment. Data represent at least 150 replication tracts from two independent experiments. Lines represent the median for each set of measurements. Boxes represent 25th and 75th quartiles with Tukey whiskers (see methods for details). Source data are provided as a source data file

treatment with mirin $(0.84 \pm 16 \mu \mathrm{m})$, by expression of hsRAD51WT $(0.85 \pm 0.15 \mu \mathrm{m})$ and by expression of hsRAD51-II3A $(0.81 \pm 0.19 \mu \mathrm{m})$. Taken together, these data indicate expression of hsRAD51-II3A rescues MRE11-dependent degradation in HR mutants with destabilized RAD51 filaments.

hsRAD51-II3A replication tracts undergo degradation by DNA2. MRE11-dependent degradation in human cells can be prevented by inhibiting replication fork regression or by stabilizing RAD51 filaments on regressed forks $7,13,15-17$. To distinguish between these two possibilities, we examined replication tract lengths in hsRAD51-II3A expressing cells after prolonged $\mathrm{HU}$ treatment. Prolonged exposure to $\mathrm{HU}(4 \mathrm{mM} \mathrm{HU}$ for $8 \mathrm{~h}$ ) was previously shown to result in nascent strand degradation by the nuclease DNA2 in U2OS cells without manipulations that decrease RAD51 levels or filament stability ${ }^{6}$. Under these conditions, DNA2dependent degradation is dependent on RAD51-mediated fork regression ${ }^{6}$. As indicated by a previous study ${ }^{6}$, the ability of DNA2 to degrade forks after prolonged $\mathrm{HU}$ treatment can be used as a readout of efficient fork regression in $\mathrm{U} 2 \mathrm{OS}$ cells ${ }^{8,11}$.

We examined fork degradation by pulsing cells with CldU followed by IdU before treatment with HU for $8 \mathrm{~h}$ and measured the ratio of IdU to CldU (Fig. 3a). In both siNS and hsRAD51WT expressing cells, prolonged $\mathrm{HU}$ treatment resulted in a significant reduction in the CldU:IdU ratio $(0.68 \pm 0.26$ (mean \pm STD) $\mu \mathrm{m}$ to $0.48 \pm 0.20 \mu \mathrm{m}$ for siNS $p$ value $<0.005,0.71 \pm 0.22$ $\mu \mathrm{m}$ to $0.46 \pm 0.20 \mu \mathrm{m}$ for hsRAD51-WT $p$ value $<0.005$, Mann-Whitney test). Reducing DNA2 expression restored ratios to those observed in untreated samples. In contrast, treatment with mirin did not result in a significant change in the CldU:IdU ratio in either siNS or hsRAD51-WT expressing cells (Fig. 3a, Supplementary Fig. 2b). These results confirm DNA2, and not MRE11, is responsible for degradation under these conditions in U2OS cells. Depletion of RAD51 did not result in tract degradation under any treatment condition. These findings confirm that RAD51 remodels HU stalled replication forks to provide a substrate for DNA2-mediated degradation in U2OS cells ${ }^{6}$. hsRAD51-II3A expressing cells exhibited a significantly reduced CldU:IdU ratio $(0.75 \pm 0.62 \mu \mathrm{m}$ to $0.62 \pm 0.30 \mu \mathrm{m}$, $p$ value $<0.005)$ that was rescued by depletion of DNA2. Treatment with mirin has no effect on the CldU:IdU ratio in hsRAD51-II3A expressing cells. We obtained an equivalent result when we measured total tract length after pulsing cells with CldU (Supplementary Fig. 2c). These results confirm that, in contrast to expectation $^{4}$, the enzymatic activity of RAD51 is not required to remodel stalled replication forks to a form that is sensitive to DNA2-mediated degradation.

Several proteins have been shown to catalyze fork regression in response to hydroxyurea including the SNF2 family translocases and the helicase $\mathrm{FBH} 1^{16,40,41}$. To determine if $\mathrm{FBH} 1$ functions in hsRAD51-II3A expressing cells to mediate fork regression, we examined replication tract lengths in hsRAD51-II3A expressing cells depleted of FBH1 after prolonged HU treatment (Fig. 3b, Supplementary Fig. 2d). As before, treatment of siNS transfected cells with HU reduced the CldU:IdU ratio $(0.89 \pm 0.22 \mu \mathrm{m}$ to 0.56 $\pm 0.17, p<0.0001)$. Depletion of FBH1 restored tract length to those observed in untreated controls $(0.82 \pm 0.17 \mu \mathrm{m})$ indicating FBH1 promotes DNA2-dependent degradation. As above, we did not observe degradation in siRAD51 transfected cells under any conditions. HU treatment of hsRAD51-WT $(0.90 \pm 0.22 \mu \mathrm{m}$ to $0.52 \pm 0.15, p<0.0001)$ and hsRAD51-II3A $(0.86 \pm 0.17 \mu \mathrm{m}$ to $0.54 \pm 0.15, p<0.0001)$ reduced the replication tract length and FBH1-depletion restored replication tract length in both cell lines. Thus, FBH1 promotes DNA2-dependent degradation in cells lacking RAD51 enzymatic activity, but this activity of FBH1 depends on the DNA binding activity of RAD51.

hsRAD51-II3A is defective in replication fork restart. Previous studies showed RAD51 is required for restart of replication forks stalled by HU treatment ${ }^{19}$. Thus, we determined if the enzymatic 
a
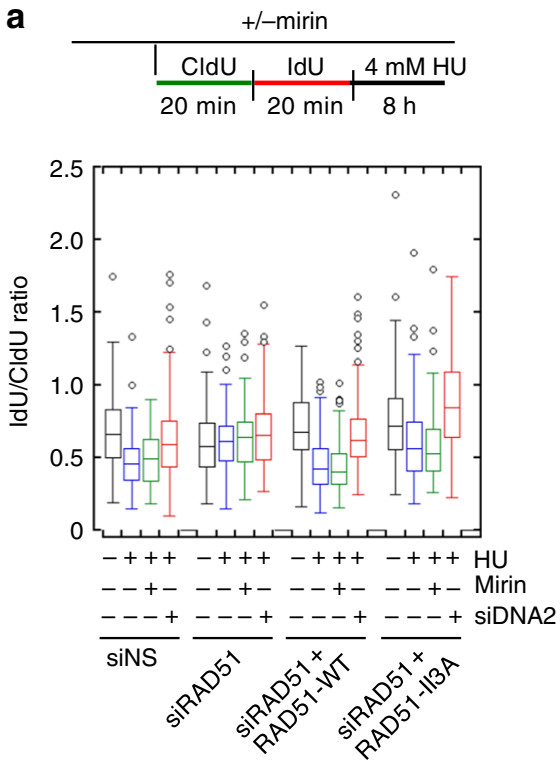

b

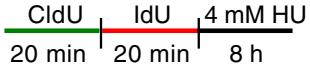

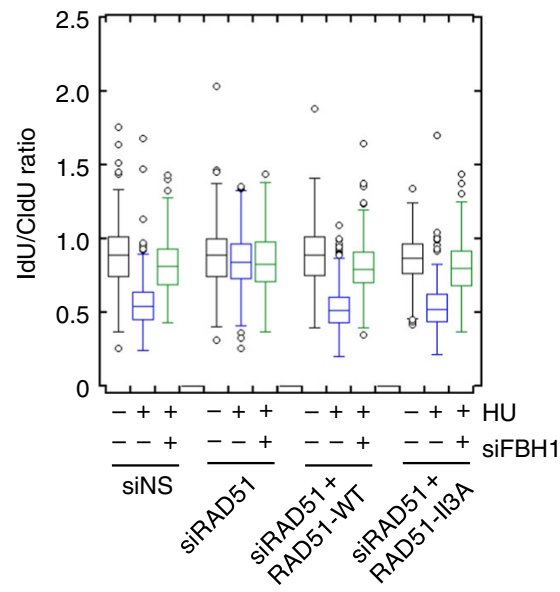

Fig. 3 Replication tract shortening after HU treatment requires DNA2 and FBH1 activity. a DNA2 depletion prevents replication tract shortening after treatment with $4 \mathrm{mM} \mathrm{HU}$ for $8 \mathrm{~h}$. Box plot represents the IdU/CldU ratio of tracts measured in U2OS cells after the indicated treatment. $\mathbf{b}$ FBH1depletion prevents replication tract shortening after treatment with $4 \mathrm{mM} \mathrm{HU}$ for $8 \mathrm{~h}$. Data represent at least 100 replication tracts from two independent experiments. Box plot represents the IdU/CldU ratio of tracts measured in U2OS cells after the indicated treatment. Lines represent the median for each set of measurements. Boxes represent 25th and 75th quartiles with Tukey whiskers (see methods for details). Values are presented as mean \pm SD. Statistical significance was determined using Mann-Whitney test. Source data are provided as a source data file

activity of RAD51 is required to restart stalled replication forks after treatment with $\mathrm{HU}$ for 5 and $8 \mathrm{~h}$ (Fig. 4). The siNS and hsRAD51-WT transfected cells showed significant levels of restart after both 5 and $8 \mathrm{~h}$ of HU treatment; in siNS control cells $50 \pm$ $5.5 \%$ (mean \pm SD) and $39 \pm 3.9 \%$ of replication forks restarted after 5 and $8 \mathrm{~h}$ of $\mathrm{HU}$ treatment respectively; in hsRAD51-WT transfected cells, the corresponding numbers were $48 \pm 3.3 \%$ and $40 \pm 3.9 \%$, respectively. Depletion of RAD51 resulted in a 2 -fold reduction in the frequency of restart at $5 \mathrm{~h}(24.3 \pm 4.8 \%$ fork restart; $p<0.005, t$ test) and a 2.3 -fold reduction at $8 \mathrm{~h}(17.1 \pm$ $2.4 \%$ fork restart; $p<0.005)$, confirming that RAD51 is required for efficient fork restart after HU treatment. hsRAD51-II3A expressing cells gave results that were intermediate between the positive and negative controls, with only a slight decrease in the efficiency of replication fork restart $(44 \pm 2.6 \%$; $p>0.05)$ at $5 \mathrm{~h}$ and a more severe (2.8-fold) reduction in the frequency of restart after $8 \mathrm{~h} \mathrm{HU}$ treatment $(13.8 \pm 2.7 \%$ fork restart; $p<0.005)$. Thus, RAD51's enzymatic activity is required for efficient replication restart, with a much greater requirement after $8 \mathrm{~h}$ as compared to $5 \mathrm{~h}$ of HU treatment. The results also raise the possibility that the strand exchange defective form of RAD51 can promote more restart than occurs when RAD51 levels are dramatically repressed. The alternative possibility is that hsRAD51-II3A has residual strand exchange activity in vivo, in spite of our inability to detect such activity biochemically. However, this seems unlikely because hsRAD51-II3A did not exhibit higher HR activity in vivo using the DR-GFP assay compared to RAD51-depleted cells (Fig. 1).

Next, we examined cells for new origin firing following a period of replication blockage by HU. New origin firing can be detected in the same double labeling experiments described above, by the presence of tracts containing only IdU labeling. We observed very little or no new origin firing $(<5 \%)$ in the positiveand negative-control experiments (Fig. 4). After $5 \mathrm{~h} \mathrm{HU}$ treatment, hsRAD51-II3A cells exhibited new origin firing similar to control cell lines. In contrast, hsRAD51-II3A expressing cells exhibited a 6.3 -fold higher level of new origin firing at $8 \mathrm{~h}$ as compared to siNS $(19 \pm 2.7 \% ; p<0.005)$. These data indicate that replication fork blockage by $\mathrm{HU}$ leads to more new origin firing in cells expressing hsRAD51-II3A, than in cells expressing hsRAD51-WT or in cells blocked for RAD51 expression.

After HU treatment, hsRAD51-II3A cells accumulate 53BP1 foci. Replication fork blockage by HU can lead to fork collapse, a process that creates a broken DNA end that recruits DNA break proteins including 53BP1 $1^{42-46}$. Replication stress-induced fork collapse and associated DNA break signaling have been shown to lead to the firing of new origins ${ }^{19}$. Given prior evidence for a functional association between new origin firing and fork collapse, we hypothesized that the new origin firing we observed in HU-treated hsRAD51-II3A cells is a consequence of an increased frequency of fork collapse. We, therefore, tested for evidence of collapsed fork accumulation specifically in S-phase cells by staining with 53BP1, which is known to localize to DSBs, and the replication fork specific marker PCNA ${ }^{47,48}$. After $8 \mathrm{~h}$ in $\mathrm{HU}$, the siNS, hsRAD51-WT positive controls, and the siRAD51 negative control, showed a 7 -fold increase in the average number of 53BP1 foci/cell $(20.5 \pm 9.6$ (mean $\pm \mathrm{SD}) 53 \mathrm{BP} 1$ foci/cell compared to $2.9 \pm 3.8$ foci per cell prior to HU treatment; Fig. 5). Expression of hsRAD51-II3A cells resulted in a significantly greater (11-fold) increase in 53BP1 foci after $8 \mathrm{~h}$ HU treatment $(47 \pm 0.853 \mathrm{BP} 1$ foci/cell; $p$ value $<0.005$, Mann-Whitney test) as compared to the untreated sample. In addition to being recruited at broken DNA ends formed by fork collapse, 53BP1 may form foci at DNA ends of reversed forks (i.e., the middle toe of the chicken foot) ${ }^{45}$, and is recruited to stalled forks early in the replication response ${ }^{49}$. As a means of determining if the observed 53BP1 foci that accumulate in RAD51-II3A expressing cells were due to fork collapse mediated by MUS81, we asked if the observed accumulation could be reduced or eliminated by siMUS81 (Fig. 5, Supplementary Fig. 3). Indeed, depletion of MUS81 reduced the number 53BP1 foci in siNS, siRAD51, and hsRAD51-WT expressing cells 1.8-fold $(12.3 \pm 7.3$ foci/cell; $p$ value $<0.005)$. In RAD51-II3A expressing cells, MUS81 depletion resulted in a 1.9 -fold decrease in the 

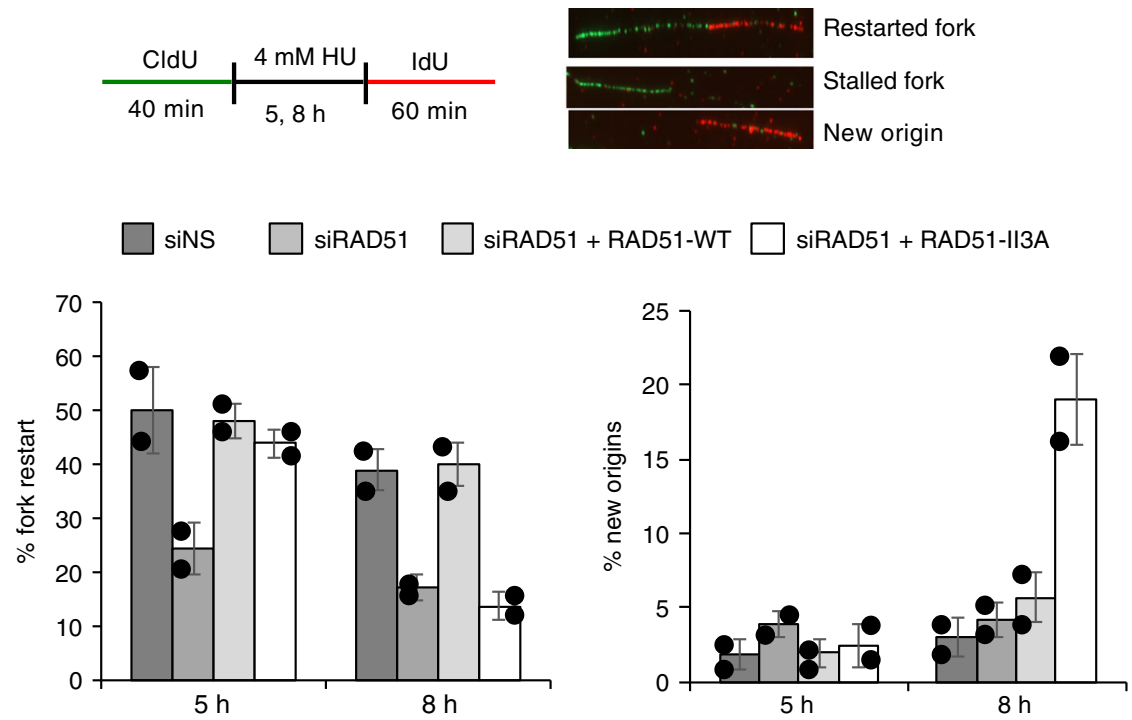

Fig. 4 Replication restart defects in hsRAD51-II3A expressing cells after treatment with HU. Graphs depict the percentage of replication forks that restart after the indicated treatments (left) or the percentage of new origin firings (right). Graph is average of two independent experiments consisting of at least 100 replication tracts with error bars representing standard deviation. Values are presented as mean \pm SD. Statistical significance was determine using a $t$ test. Schematic of experimental design is above the graphs and fibers that represent restarted forks (CldU + IdU), stalled fork (CldU only) and new origin (IdU only) are shown. Source data are provided as a source data file

number of 53BP1 foci per nucleus $(24.5 \pm 11.8$ foci/cell; $p$ value $<$ $0.005)$ providing evidence that 53BP1 focus formation is associated with MUS81-dependent cleavage. As a control against the possibility that 53BP1 activity differs between cultures, a fraction of each culture was treated with HU for $24 \mathrm{~h}$. This highly prolonged replication arrest caused equivalent induction of 53BP1 foci and MUS81 depletion significantly reduced 53BP1 foci in all samples, as expected (Supplementary Fig. 3). Together, the results suggest that hsRAD51-II3A causes more accumulation of collapsed forks following $8 \mathrm{~h}$ HU treatment than occurs in cells expressing equivalent levels of hsRAD51-WT, and also more than in cells expressing very low levels of RAD51. The possible mechanistic basis for these observations is discussed below.

\section{Discussion}

RAD51 has been implicated in several steps in the response to replication stress, including fork protection, replication fork remodeling, and replication fork restart. Here, we utilized a RAD51 mutant allele that retains DNA binding activity, but is defective in strand exchange to gain mechanistic insight into the role of RAD51's enzymatic activity at stalled replication forks. Previous studies have suggested that stabilization of RAD51 filaments is sufficient to protect from MRE11-dependent degradation ${ }^{7,13}$. This study utilized the RAD51-K133R mutant that is defective for HR-mediated repair, but has robust strand exchange activity precluding the use of this mutant to definitively determine if RAD51 DNA binding activity alone is sufficient to protect from MRE11-dependent degradation ${ }^{26,27}$. We found that the ability of RAD51 to protect nascent strands from MRE11mediated degradation is independent of strand exchange activity in both BRCA2- and FANCD2-deficient cell lines supporting the hypothesis that RAD51 filament formation is sufficient to protect replication forks from pathological degradation. Our results provide additional insight into the mechanism of RAD51dependent replication fork remodeling by showing that degradation of regressed forks by DNA2 does not require RAD51's enzymatic activity. We further show that the enzymatic activity of RAD51 is required for efficient replication restart after prolonged HU exposure.
Prolonged HU treatment of cells results in DNA2-dependent degradation that is not dependent on BRCA2-RAD51 fork protection, but depends on RAD51-dependent replication fork regression $^{6,11}$. We provide evidence that the enzymatic activity of RAD51 is not required for DNA2-dependent degradation of stalled forks under conditions of prolonged replication stress suggesting that DNA binding activity of RAD51 is sufficient to promote replication fork regression. How can the DNA binding activity of RAD51 promote replication fork regression? RAD51 interacts with polymerase a preventing the formation of ssDNA gaps at stalled forks ${ }^{16}$. If annealing of complementary nascent strands is important to drive fork regression, RAD51 preventing significant ssDNA formation at the fork may be sufficient to drive fork regression. Alternatively, DNA-bound RAD51 could promote fork regression by recruiting other proteins that act directly to catalyze the process. RAD54 50 , FANCM ${ }^{51}$, HTLF $^{52}$, and ZRANB3 ${ }^{53,54}$, have been found to be able to reverse a model replication fork substrate in vitro and $\mathrm{FBH}_{1}{ }^{40}$, SMARCL $1^{15}$, and ZRANB3 $^{41}$ have been shown to promote fork regression in vivo. Here, we show FBH1 depletion in RAD51-II3A expressing cells prevents DNA2-dependent degradation, providing evidence for a pathway of fork regression that depends on both the nonenzymatic activity of RAD51 and on FBH1. Importantly, we see no difference in the level of DNA2 sensitivity in cells blocked for FBH1 expression when cells expressing RAD51-WT or RAD51II3A are compared. This finding implies that the enzymatic activity of RAD51 cannot substitute for FBH1 in promoting fork regression under these conditions. We cannot rule out the possibility that RAD51 enzymatic activity is capable of promoting fork regression in other types of human cells, but that strand exchangeindependent mechanisms involving fork remodeling proteins such as SMARCL1, ZRANB3, and FBH1 predominate in U2OS cells.

hsRAD51-II3A cells promoted significant restart after $5 \mathrm{~h} \mathrm{HU}$ treatment, but were highly defective in replication restart after longer $(8 \mathrm{~h})$ treatment with HU. Together, our results lead us to a model for three distinct pathways to restart stalled replication forks, one that is RAD51-dependent, strand exchange-dependent; a second that is RAD51-dependent, strand exchange-independent; and a third that is RAD51-independent. Further, our results 

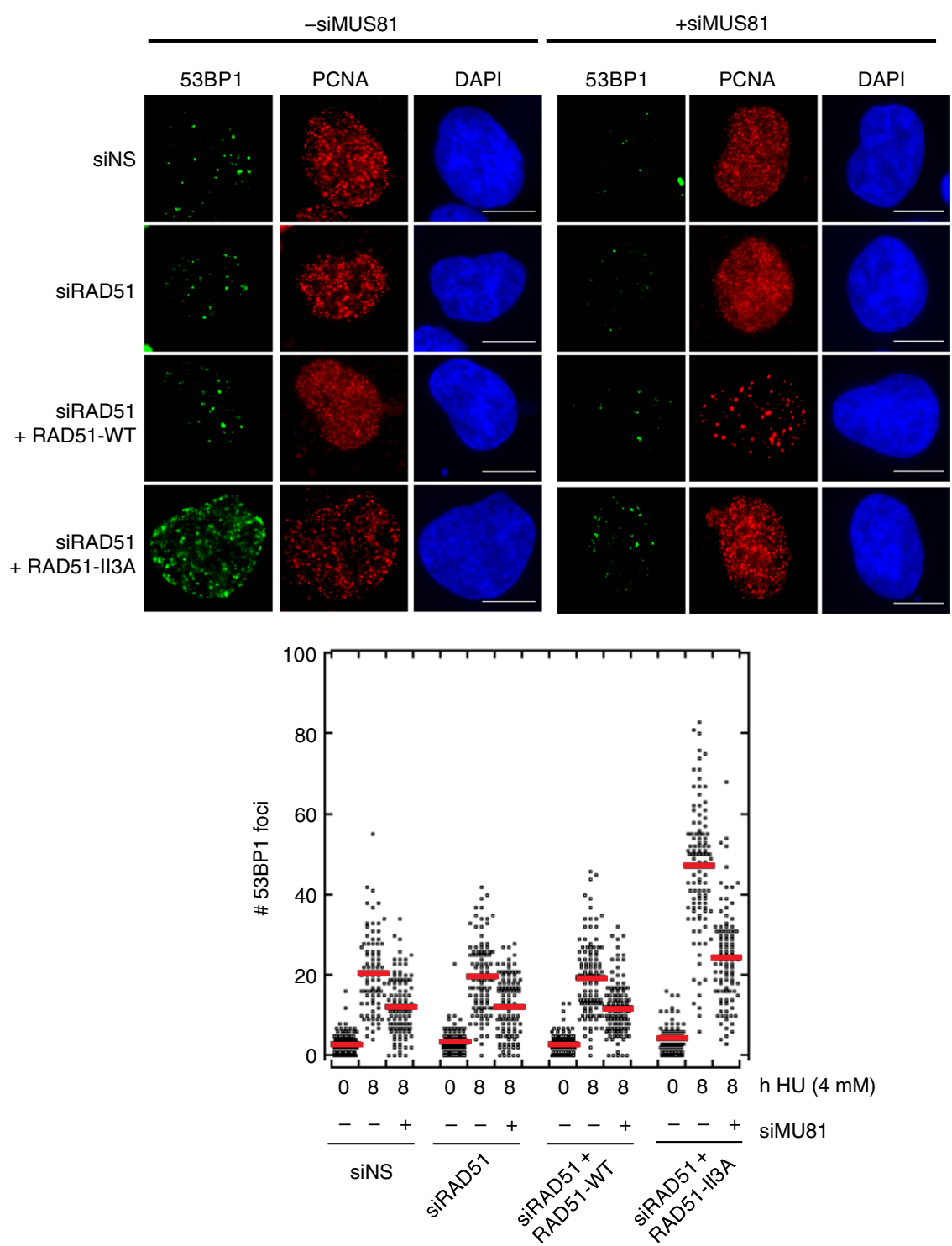

Fig. 5 hsRAD51-II3A expressing cells accumulate 53BP1 foci after treatment with HU. Representative images depicting 53BP1 foci (green) in PCNA (red) positive cells after the indicated treatments. DNA is stained in blue. Scale bar $=10 \mu \mathrm{m}$. Dot plot depicts quantitation of 53BP1 foci after indicated treatments. Data is combined data consisting of at least 100 nuclei from two independent experiments. Red line represents the mean. Values are presented as mean \pm SD. Statistical significance was determined using the Mann-Whitney $U$ test. Source data are provided as a source data file

indicate that the RAD51-dependent, strand exchange-dependent mechanism is more predominant after $8 \mathrm{~h}$ of exposure to $\mathrm{HU}$ as compared to $5 \mathrm{~h}$ of exposure, while the converse is true for the RAD51-dependent, strand exchange-independent mechanism. These data are consistent with a previous study that identified an early, cleavage-independent restart pathway involving 53BP1 and a late, HR-dependent pathway involving BRCA1 ${ }^{49}$. Further studies are required to determine if the role of RAD51 early in the replication stress response depends on 53BP1 or if this pathway represents the RAD51-independent pathway identified by our results. Our results are also consistent with work using an allele of Schizosaccharomyces pombe rad51 that was modeled on $S$. cerevisiae Rad51-II3A, but not biochemically characterized. That work led to the proposal that strand exchange activity coded by $S$. pombe rad51 $1^{+}$is dispensable for replication fork protection from Exo1, but required for efficient fork restart ${ }^{55}$.

Combining all the data, we propose the following model for RAD51-dependent replication fork remodeling and restart (Fig. 6). At early times after fork blockage, binding of RAD51 to DNA is sufficient to protect the replisome by preventing excessive uncoupling of the replication fork; thereby preventing significant ssDNA accumulation. After fork regression and processing of regressed arms by MRE11, EXO1, and/or DNA2, RAD51 loading onto the regressed arm prevents pathological degradation by nucleases. When the replication block is removed, reversed forks can be resolved by the action of helicases such as RECQ1, reinstating the replication fork ${ }^{56}$. In contrast, prolonged stalling of a replication fork results in the formation of an intermediate that requires the strand exchange activity of RAD51 for restart. One possibility is that MRE11and DNA2-mediated resection of the middle toe of the reversed fork provides a single-stranded overhang that serves as a substrate for formation of a RAD51 nucleoprotein filament. In this instance, RAD51-mediated strand invasion is used to reinstate the replication fork. Alternatively, endonucleolytic cleavage of reversed fork intermediates by nucleases such as MUS81 and SLX1 may form collapsed fork structures containing singleended DNA breaks ${ }^{57,58}$. These structures are expected to require RAD51-mediated strand exchange to restore functional forks $^{19}$. Consistent with this, hsRAD51-II3A expressing cells 


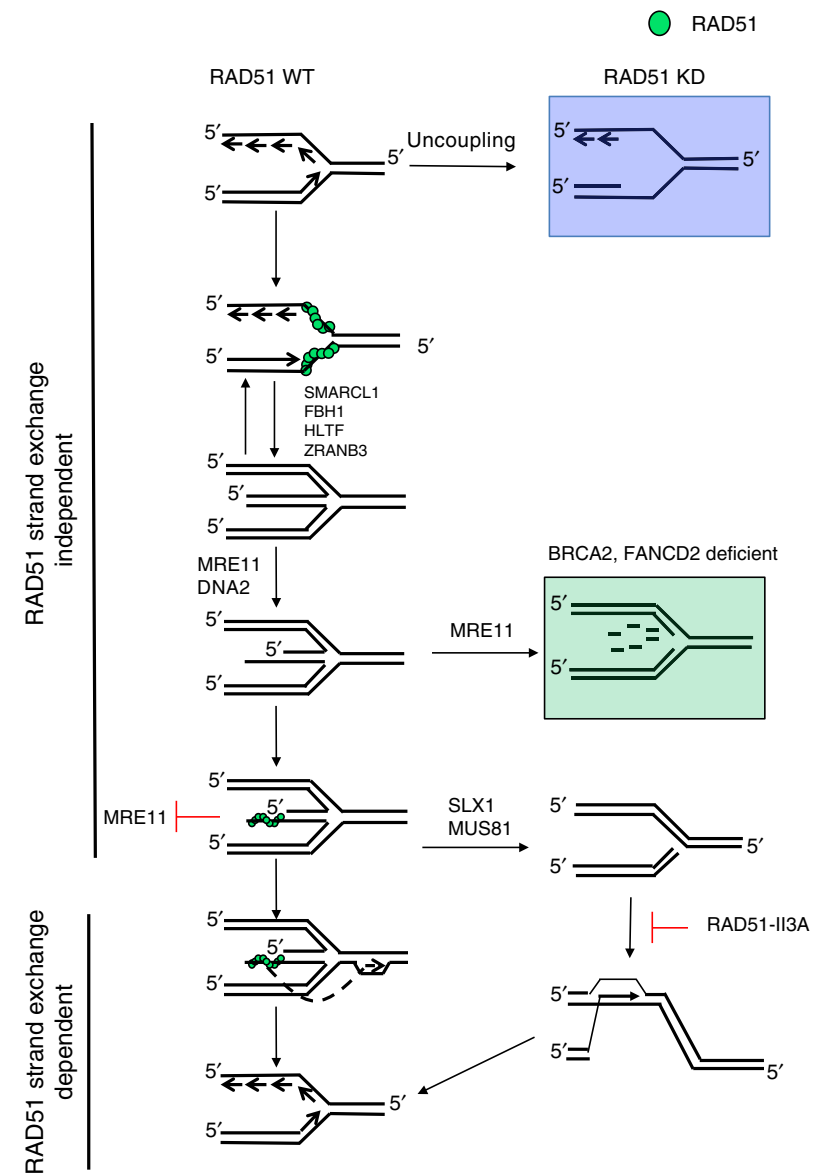

Fig. 6 Model depicting role of RAD51 at stalled replication forks. RAD51 binds to a stalled replication fork and stabilizes it by preventing extensive ssDNA formation. Proteins such as SMARCL1 and FBH1 promote replication fork regression resulting in the formation of the chicken foot structure. DNA2 resects the middle toe providing a substrate for RAD51 strand exchange-dependent fork restart. DNA2 also promotes degradation of nascent strands after fork regression in cells expressing hsRAD51-WT or hsRAD51-II3A (not depicted). In RAD51-depleted cells (RAD51 KD), the replication fork contains excess SsDNA due to uncoupling (Blue Box). hsRAD51-II3A is able to prevent MRE11-degradation by binding directly to the middle toe. hsRAD51-II3A is unable to restart the fork via strand exchange activity resulting in trapped replication intermediate that often consists of a one-ended DSB formed by MUS81-dependent cleavage of a reversed fork. The one-ended DSB formed requires RAD51 strand exchange activity to repair the break and reinstate the replication fork

accumulate 53BP1 foci that are dependent on MUS81 activity, indicating that a substantial fraction of the accumulated foci represents collapsed replication forks formed by MUS81 cleavage. hsRAD51-II3A expressing cells also exhibit increased origin firing. These phenotypes are associated with the accumulation of collapsed replication forks ${ }^{19}$. Interestingly, the collapsed fork-associated phenotypes observed in hsRAD51II3A expressing cells are more severe than those observed in RAD51-depleted cells. This observation suggests that replication fork remodeling mediated by hsRAD51-II3A traps replication intermediates that cannot be resolved by RAD51 strand exchange-independent pathways. The partial dependency of 53BP1 foci on MUS81 suggests that a major fraction of the trapped intermediates are collapsed forks, but it is possible that the processing of unbroken reversed forks is also blocked by RAD51-II3A, given prior evidence that 53BP1 can localize to replication forks prior to DSB formation ${ }^{45,49}$.
Here, we demonstrate RAD51 DNA binding activity alone is sufficient for replication fork protection and regression, but strand exchange activity is required for a significant fraction of replication fork restart. Future work will determine precisely what types of replication-associated structures require the strand exchange activity of RAD51. It will also be of interest to determine if strand exchange activity of RAD51 has additional roles at replication forks under conditions which require repair of a physical lesion (e.g., interstrand cross-links), or conditions that only result in a moderate reduction in replication fork speed (e.g., UV-light induced damage) $)^{10,18}$.

\begin{abstract}
Methods
Purification of hsRAD51-WT and hsRAD51-II3A mutant. The open reading frames of hsRAD51-WT and hsRAD51-II3A mutant with a C-terminal His-6 tag were cloned into pET21d (Novagen). The proteins were overexpressed in Escherichia coli Rosetta(DE3) plysS cells by induction using $0.5 \mathrm{mM}$ IPTG at $37^{\circ} \mathrm{C}$ for 3 h. All the following steps for purification were performed at $4{ }^{\circ} \mathrm{C}$. Bacterial lysates with expressed human RAD51 variants were generated by French press and cleared by ultracentrifugation at 120,000 $\times \mathrm{g}$ for $30 \mathrm{~min}$. The human RAD51 protein variants were then purified from the cleared lysates in sequential steps using nickel columns (GE Healthcare) by gravity, followed by His-Trap FF in FPLC (GE Healthcare). The eluted protein fractions were analyzed by $12 \%$ sodium dodecyl sulphate polyacrylamide gel electrophoresis. The purified protein fractions were pooled, concentrated by Amicon (Millipore), and exchanged into storage buffer ( $25 \mathrm{mM}$ Tris-HCl, pH 7.5, $1 \mathrm{mM}$ DTT, $250 \mathrm{mM} \mathrm{NaCl}_{2}, 0.5 \mathrm{mM}$ EDTA, $10 \%$ glycerol) by PD10 column (Sephadex G25, GE Healthcare).
\end{abstract}

DNA-binding assay. The binding of hsRAD51 to ssDNA was assayed by the FP method in $50 \mu$ l. The ssDNA used was an 84-mer conjugated with Alexa Flour-488 at its $5^{\prime}$ end (5'GGTAGCGGTTGGGTGAGTGGTGGGGAGGGTCGGGAGGTG GCGTAGAAACATGAT AGGAATGTGAATGAATGAAGTACAAGTAAA-3'; synthesized by Integrated DNA Technologies) and was used at $200 \mathrm{nM}$ nucleotides $(2.4 \mathrm{nM})$. Human Rad51 concentration range was from 10 to $1000 \mathrm{nM}$. The binding reactions were in buffer $\mathrm{B}\left(25 \mathrm{mM}\right.$ Tris- $\mathrm{HCl}$ (pH 7.8), $1 \mathrm{mM} \mathrm{MgCl}_{2}, 1 \mathrm{mM}$ ATP, $1 \mathrm{mM}$ DTT, $50 \mathrm{mM} \mathrm{NaCl}_{2}, 50 \mu \mathrm{M} \mathrm{CaCl}_{2}$, and $100 \mu \mathrm{g} / \mathrm{ml}$ bovine serum albumin (BSA)). The reactions were initiated by adding protein to ssDNA and incubated at $37^{\circ} \mathrm{C}$ for $30 \mathrm{~min}$. The $\mathrm{FP}$ (in $\mathrm{mP}$ units) was measured using a Tecan Infinite F200 PRO plate reader. All binding conditions were performed in triplicate, and the mean values were plotted with standard error of the mean. Buffer and ssDNA had no effect on FP in the absence of added protein; any background FP from buffer or ssDNA has been subtracted from the corresponding values containing proteins.

D-loop assay. The assay was performed in $10 \mu$ in a buffer containing $25 \mathrm{mM}$ Tris- $\mathrm{HCl}$ (pH 7.8); $1 \mathrm{mM} \mathrm{MgCl} 2,1 \mathrm{mM}$ ATP, $1 \mathrm{mM}$ DTT, $50 \mu \mathrm{M} \mathrm{CaCl}_{2}$, and 100 $\mu \mathrm{g} / \mathrm{ml}$ BSA. ${ }^{32} \mathrm{P}-90 \mathrm{mer}$ ssDNA (5'TACGAATGCACACGGTGTGGTGGGC CCAGGTATTGTTAGCGGTTTG AAGCAGGCGGCAGAAGAAGTAACAAAG GAACCTAGAGGCCTTTT) was used at $3.6 \mu \mathrm{M}$ nucleotide or $40 \mathrm{nM}$; negative

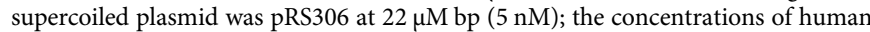
RAD51 protein variants were $0.5,1,1.5$, and $2 \mu \mathrm{M}$ and as indicated in Fig. 1b. In the assay, human RAD51 was first incubated with ssDNA at $37^{\circ} \mathrm{C}$ for $10 \mathrm{~min}$, followed by the addition of $1 \mu \mathrm{l}$ of supercoiled plasmid to initiate D-loop formation; the mixture was incubated further at $37^{\circ} \mathrm{C}$ for $15 \mathrm{~min}$. The reaction was stopped by adding a $2 \mu \mathrm{l}$ mixture of sodium dodecyl sulphate $(1 \% \mathrm{w} / \mathrm{v})$ and protease $\mathrm{K}(1 \mathrm{mg} /$ $\mathrm{ml}$ ) followed by incubation at $37^{\circ} \mathrm{C}$ for $5 \mathrm{~min}$ for deproteinization. The samples were analyzed by electrophoresis in $1 \%$ agarose gel in $1 \times$ TAE buffer. The gel was dried onto a positively charged nylon membrane (Millipore), exposed to an image plate, and analyzed using the Typhoon 9200 Phosphoimager (GE Healthcare) and the computer software Quantity One. Quantification of radioactive image intensities was performed with data within a linear range of exposure. The D-loop yield was expressed as a percentage of input plasmid.

Cell culture. U2OS DR-GFP cells contain a stably integrated DR-GFP assay to measure HR and was generated by the lab of Maria Jasin ${ }^{59}$. Cells were grown in DMEM (Gibco) supplemented with $10 \%$ fetal bovine serum. The authenticity of the cell line was validated previously by short tandem repeat profiling at the Genetic Resources Core Facility at John Hopkins School of Medicine (Baltimore, $\mathrm{MD})^{60}$. Cell line was monitored monthly for mycoplasma contamination.

Expression of RAD51 in U20S cells. WT RAD51 or RAD51 cDNA containing mutations in the secondary binding site (R130A, R303A, and K313A) was cloned into pcDNA 3.1 (Invitrogen) using Gibson assembly per manufacturer's instructions (New England Biolabs).

The following primers were used to amplify the fragments used for cloning. For amplification of RAD51 cDNA: 
51_pcDNA_F 5' tagcgtttaaacttaagcttGAATTGATCCATGGCAATG 51_pcDNA_R 5'ctagactcgagcggccgccaCCCAATGATTCAGTCTTTG For amplification of pcDNA 3.1 vector:

pcDNA_F 5' TGGCGGCCGCTCGAGTCT

pcDNA_R 5' AAGCTTAAGTTTAAACGCTAGCCAGCTTGG

U2OS cells were transfected with pcDNA3.1, hsRAD51-WT (pNRB707), or hsRAD51-II3A (pNRB708) expression plasmids using Lipofectamine 3000 (Invitrogen) as per manufacturer's instructions. After $24 \mathrm{~h}$, cells were transfected with RAD51 siRNAs targeting the 3'UTR. At 48-hours post transfection, cells were collected and analyzed for the various assays.

siRNA sequences. siRNAs were transfected using Lipofetamine RNAiMAX as per manufacturer's instructions (Invitrogen). The All-Star negative control (siNS) siRNA was used as a control (Qiagen). The following siRNA sequences were used in this study.

siRAD51 5' GACUGCCAGGAUAAAGCUU was used in a previous study ${ }^{61}$. siDNA2 5' CAGUAUCUCCUCUAGCUAG was used in a previous study ${ }^{6}$. siFANCD2 $5^{\prime}$ CAGAGUUUGCUUCACUCUCUA was used in a previous study ${ }^{62}$ siMUS81 5' CAGCCCUGGUGGAUCGAUA was used in a previous study ${ }^{6}$. siFBH1 5' GGAUGUUUGCAAGAGAGUCAGGAAA.

Nascent DNA fiber assay. Cells were pulsed with CldU $(50 \mu \mathrm{M})$, or CldU $(50 \mu \mathrm{M})$ followed by IdU $(150 \mu \mathrm{M})$ and treated with $\mathrm{HU}(4 \mathrm{mM})$ for the indicated times. To measure replication restart, cells were pulsed with CldU $(50 \mu \mathrm{M})$ before treatment with $4 \mathrm{mM} \mathrm{HU}$ for the indicated times. HU was removed and cells were pulsed with IdU $(50 \mu \mathrm{M})$. Mirin $(50 \mu \mathrm{M})$ was added 30 min prior to the pulse with CldU and was present throughout the experiment. DNA fibers were prepared by spotting $200-400$ cells onto the slide. Cells were lysed and DNA fibers were spread by tilting slides. After drying for $1 \mathrm{~h}$, slides were fixed with 3:1 methanol:glacial acetic acid and allowed to dry overnight. Slides were denatured in $2.5 \mathrm{M}$ hydrochloric acid before staining with antibodies to CldU and IdU. The primary antibodies used were mouse anti-BrdU/IdU (1:50; B44, BD Biosciences) and anti-BrdU/CldU (1:400, ab6326, Abcam) followed by staining with Alexa Fluor conjugated secondary antibodies (ThermoScientific, 1:1000). Tract lengths were measured using Image J. The box plots represent pooled data from at least two independent experiments. Box Plots were generated using Kaleidagraph software. Whiskers are generated using the Tukey's method. First, the interquartile range (IQR) is calculated (the difference between the 25th and 75th percentiles). The whiskers represent the 75th percentile plus 1.5 times IQR and the 25th percentile minus 1.5 times IQR. Statistical significance was determined using Mann-Whitney test.

RAD51 and 53BP1 focus formation. Forty-eight hour after transfection with siRNAs, cells were treated with $4 \mathrm{mM} \mathrm{HU}$ for the indicated times. For RAD51 focus formation, cells were treated with $6 \mathrm{~Gy}$ using a maxitron $\mathrm{X}$-ray generator. Cells were pre-extracted with HEPES/Triton-X buffer (20 mM HEPES, ph7.4, 0.5\% Triton-X 100, $50 \mathrm{mM} \mathrm{NaCl}, 3 \mathrm{mM} \mathrm{MgCl}, 300 \mathrm{mM}$ sucrose in water) and fixed with $3 \%$ paraformaldehyde, $3.4 \%$ sucrose in $\mathrm{PBS}^{34}$. Cells were washed in PBS and stained with the indicated primary antibodies, washed and stained with Alexa Fluor conjugated secondary antibodies (1:1000, ThermoScientific). Images were acquired at $100 \times$ magnification with a Zeiss upright M2 epifluorescence microscope. Antibodies used in this study are as followed: RAD51 is a rabbit polyclonal antibody against purified human RAD51 (1:1000, Pacific Immunology). 53BP1 (1:1000, NB100-304) was from Novus Biologicals and PCNA (1:1000, IG7) was from Abnova. Dot plots represent combined data from two independent experiments. Statistical significance was determined by the Wilcoxon Rank Sum Test.

Western blotting. Western blotting was performed using standard methods. AntiDNA2 (1:500; ab96488), anti-MUS81 (1:1000, ab14387), and anti-FBH1 (1:100, ab58881) were from Abcam. Anti-FANCD2 (1:1000, NB100-182) was from Novus Biologicals. Proteins were detected using a C-DIGIT blot scanner (Licor). All uncropped blots are included in the source data file.

DR-GFP assay. U2OS cells containing the DR-GFP construct stably integrated into the genome were transfected with a plasmid expressing I-SceI (pBAS) or an empty vector (pCAGG) after the indicated treatment ${ }^{38}$. After $48 \mathrm{~h}$, cells were collected and the percentage of cells expressing GFP was determined by flow cytometry (LSR II, BD Biosciences). At least 20,000 cells were counted. Gated strategy is illustrated in Supplementary Fig. 4. Statistical significance was determined using a two-tailed $t$ test.

Statistical analysis. All the data are presented as averages of at least two independent experiments. Dot plots represent pooled data. Statistical analysis between samples were performed by one-sided analysis of variance (ANOVA) (DR-GFP, replication fork restart), student $t$ test (DR-GFP, replication fork restart), and Mann-Whitney test (replication tracts, focus counts). Tests were performed using DNA Prism software or built-in tools in Kaleidagraph. $p<0.05$ was considered significant and relevant $p$ values are reported in the text. The statistical test is reported in the figure legends.
Reporting summary. Further information on research design is available in the Nature Research Reporting Summary linked to this article.

\section{Data availability}

Source data are provided as a source data file. All data supporting the findings of this study are available from the corresponding authors upon request.

Received: 17 April 2018 Accepted: 22 August 2019

Published online: 27 September 2019

\section{References}

1. Kolinjivadi, A. M. et al. Moonlighting at replication forks-a new life for homologous recombination proteins BRCA1, BRCA2 and RAD51. FEBS Lett. 47, 222 (2017).

2. Berti, M. \& Vindigni, A. Replication stress: getting back on track. Nat. Struct. Mol. Biol. 23, 103-109 (2016).

3. San Filippo, J., Sung, P. \& Klein, H. Mechanism of eukaryotic homologous recombination. Annu. Rev. Biochem. 77, 229-257 (2008).

4. Zellweger, R. et al. Rad51-mediated replication fork reversal is a global response to genotoxic treatments in human cells. J. Cell Biol. 208, 563-579 (2015).

5. Mijic, S. et al. Replication fork reversal triggers fork degradation in BRCA2defective cells. Nat. Commun. 8, 859 (2017).

6. Thangavel, $\mathrm{S}$. et al. DNA2 drives processing and restart of reversed replication forks in human cells. J. Cell Biol. 208, 545-562 (2015).

7. Schlacher, K. et al. Double-strand break repair-independent role for BRCA2 in blocking stalled replication fork degradation by MRE11. Cell 145, 529-542 (2011).

8. Spies, J. et al. Nek1 regulates Rad54 to orchestrate homologous recombination and replication fork stability. Mol. Cell 62, 903-917 (2016).

9. Su, F. et al. Nonenzymatic role for WRN in preserving nascent DNA strands after replication stress. Cell Rep. 9, 1387-1401 (2014).

10. Vallerga, M. B., Mansilla, S. F., Federico, M. B., Bertolin, A. P. \& Gottifredi, V. Rad51 recombinase prevents Mre11 nuclease-dependent degradation and excessive PrimPol-mediated elongation of nascent DNA after UV irradiation. Proc. Natl Acad. Sci. USA 112, E6624-33 (2015).

11. Bhat, K. P. et al. RADX modulates RAD51 activity to control replication fork protection. Cell Rep. 24, 538-545 (2018).

12. Hashimoto, Y., Ray Chaudhuri, A., Lopes, M. \& Costanzo, V. Rad51 protects nascent DNA from Mre11-dependent degradation and promotes continuous DNA synthesis. Nat. Struct. Mol. Biol. 17, 1305-1311 (2010).

13. Schlacher, K., Wu, H. \& Jasin, M. A distinct replication fork protection pathway connects Fanconi anemia tumor suppressors to RAD51-BRCA1/2. Cancer Cell 22, 106-116 (2012).

14. Somyajit, K., Saxena, S., Babu, S., Mishra, A. \& Nagaraju, G. Mammalian RAD51 paralogs protect nascent DNA at stalled forks and mediate replication restart. Nucleic Acids Res. 43, 9835-9855 (2015).

15. Taglialatela, A. et al. Restoration of replication fork stability in BRCA1- and BRCA2-deficient cells by inactivation of SNF2-family fork remodelers. Mol. Cell 68, 414-430.e8 (2017).

16. Kolinjivadi, A. M. et al. Smarcal1-mediated fork reversal triggers Mre11dependent degradation of nascent DNA in the absence of Brca2 and stable Rad51 nucleofilaments. Mol. Cell 67, 867-881.e7 (2017)

17. Lemaçon, D. et al. MRE11 and EXO1 nucleases degrade reversed forks and elicit MUS81-dependent fork rescue in BRCA2-deficient cells. Nat. Commun. 8, 860 (2017).

18. Wang, A. T. et al. A dominant mutation in human RAD51 reveals its function in DNA interstrand crosslink repair independent of homologous recombination. Mol. Cell 59, 478-490 (2015).

19. Petermann, E., Orta, M. L., Issaeva, N., Schultz, N. \& Helleday, T. Hydroxyurea-stalled replication forks become progressively inactivated and require two different RAD51-mediated pathways for restart and repair. Mol. Cell 37, 492-502 (2010).

20. Hanada, K. et al. The structure-specific endonuclease Mus81 contributes to replication restart by generating double-strand DNA breaks. Nat. Struct. Mol. Biol. 14, 1096-1104 (2007).

21. Franchitto, A. et al. Replication fork stalling in WRN-deficient cells is overcome by prompt activation of a MUS81-dependent pathway. J. Cell Biol. 183, 241-252 (2008).

22. Pepe, A. \& West, S. C. MUS81-EME2 promotes replication fork restart. Cell Rep. 7, 1048-1055 (2014).

23. Hashimoto, Y., Puddu, F. \& Costanzo, V. RAD51- and MRE11-dependent reassembly of uncoupled CMG helicase complex at collapsed replication forks. Nat. Struct. Mol. Biol. 19, 17-24 (2011). 
24. Trenz, K., Smith, E., Smith, S. \& Costanzo, V. ATM and ATR promote Mre11 dependent restart of collapsed replication forks and prevent accumulation of DNA breaks. EMBO J. 25, 1764-1774 (2006).

25. Ray Chaudhuri, A. et al. Replication fork stability confers chemoresistance in BRCA-deficient cells. Nature 535, 382-387 (2016).

26. Chi, P., Van Komen, S., Sehorn, M. G., Sigurdsson, S. \& Sung, P. Roles of ATP binding and ATP hydrolysis in human Rad51 recombinase function. DNA Repair 5, 381-391 (2006).

27. Morrison, C. et al. The essential functions of human Rad51 are independent of ATP hydrolysis. Mol. Cell. Biol. 19, 6891-6897 (1999).

28. Forget, A. L., Loftus, M. S., McGrew, D. A., Bennett, B. T. \& Knight, K. L. The human Rad51 K133A mutant is functional for DNA double-strand break repair in human cells. Biochemistry 46, 3566-3575 (2007).

29. Hsieh, P., Camerini-Otero, C. S. \& Camerini-Otero, R. D. The synapsis event in the homologous pairing of DNAs: RecA recognizes and pairs less than one helical repeat of DNA. Proc. Natl Acad. Sci. USA 89, 6492-6496 (1992).

30. Lee, J. Y. et al. DNA RECOMBINATION. Base triplet stepping by the Rad51/ RecA family of recombinases. Science 349, 977-981 (2015).

31. Cloud, V., Chan, Y.-L., Grubb, J., Budke, B. \& Bishop, D. K. Rad51 is an accessory factor for Dmc1-mediated joint molecule formation during meiosis. Science 337, 1222-1225 (2012).

32. Chen, Z., Yang, H. \& Pavletich, N. P. Mechanism of homologous recombination from the RecA-ssDNA/dsDNA structures. Nature 453, 489-484 (2008).

33. Jayathilaka, K. et al. A chemical compound that stimulates the human homologous recombination protein RAD51. Proc. Natl Acad. Sci. USA 105 15848-15853 (2008).

34. Mason, J. M. et al. RAD54 family translocases counter genotoxic effects of RAD51 in human tumor cells. Nucleic Acids Res. 43, 3180-3196 (2015)

35. Haaf, T., Golub, E. I., Reddy, G., Radding, C. M. \& Ward, D. C. Nuclear foci of mammalian Rad51 recombination protein in somatic cells after DNA damage and its localization in synaptonemal complexes. Proc. Natl Acad. Sci. USA 92, 2298-2302 (1995).

36. Raderschall, E., Golub, E. I. \& Haaf, T. Nuclear foci of mammalian recombination proteins are located at single-stranded DNA regions formed after DNA damage. Proc. Natl Acad. Sci. USA 96, 1921-1926 (1999).

37. Bishop, D. K. et al. Xrcc3 is required for assembly of Rad51 complexes in vivo. J. Biol. Chem. 273, 21482-21488 (1998).

38. Bennardo, N., Cheng, A., Huang, N. \& Stark, J. M. Alternative-NHEJ is a mechanistically distinct pathway of mammalian chromosome break repair. PLoS Genet. 4, e1000110 (2008).

39. Rondinelli, B. et al. EZH2 promotes degradation of stalled replication forks by recruiting MUS81 through histone $\mathrm{H} 3$ trimethylation. Nat. Cell Biol. 19, 1371-1378 (2017).

40. Fugger, K. et al. FBH1 catalyzes regression of stalled replication forks. Cell Rep. 10, 1749-1757 (2015).

41. Vujanovic, M. et al. Replication fork slowing and reversal upon DNA damage require PCNA polyubiquitination and ZRANB3 DNA translocase activity. Mol. Cell 67, 882-890.e5 (2017).

42. Groth, P. et al. Methylated DNA causes a physical block to replication forks independently of damage signalling, $\mathrm{O}(6)$-methylguanine or DNA single-strand breaks and results in DNA damage. J. Mol. Biol. 402, 70-82 (2010).

43. Fedor, Y. et al. From single-strand breaks to double-strand breaks during Sphase: a new mode of action of the Escherichia coli cytolethal distending toxin. Cell. Microbiol. 15, 1-15 (2013).

44. Jones, R. M., Kotsantis, P., Stewart, G. S., Groth, P. \& Petermann, E. BRCA2 and RAD51 promote double-strand break formation and cell death in response to gemcitabine. Mol. Cancer Ther. 13, 2412-2421 (2014).

45. Ray Chaudhuri, A., Ahuja, A. K., Herrador, R. \& Lopes, M. Poly(ADP-ribosyl) glycohydrolase prevents the accumulation of unusual replication structures during unperturbed S phase. Mol. Cell. Biol. 35, 856-865 (2015).

46. Ray Chaudhuri, A. et al. Topoisomerase I poisoning results in PARP-mediated replication fork reversal. Nat. Struct. Mol. Biol. 19, 417-423 (2012)

47. Bravo, R. \& Macdonald-Bravo, H. Existence of two populations of cyclin/ proliferating cell nuclear antigen during the cell cycle: association with DNA replication sites. J. Cell Biol. 105, 1549-1554 (1987).

48. Schultz, L. B., Chehab, N. H., Malikzay, A. \& Halazonetis, T. D. p53 binding protein 1 (53BP1) is an early participant in the cellular response to DNA double-strand breaks. J. Cell Biol. 151, 1381-1390 (2000).

49. $\mathrm{Xu}, \mathrm{Y}$. et al. 53BP1 and BRCA1 control pathway choice for stalled replication restart. Elife 6, 35897 (2017).

50. Bugreev, D. V., Rossi, M. J. \& Mazin, A. V. Cooperation of RAD51 and RAD54 in regression of a model replication fork. Nucleic Acids Res. 39, 2153-2164 (2011).
51. Gari, K., Décaillet, C., Delannoy, M., Wu, L. \& Constantinou, A. Remodeling of DNA replication structures by the branch point translocase FANCM. Proc. Natl Acad. Sci. USA 105, 16107-16112 (2008).

52. Kile, A. C. et al. HLTF's ancient HIRAN Domain Binds $3^{\prime}$ DNA ends to drive replication fork reversal. Mol. Cell 58, 1090-1100 (2015).

53. Ciccia, A. et al. Polyubiquitinated PCNA recruits the ZRANB3 translocase to maintain genomic integrity after replication stress. Mol. Cell 47, 396-409 (2012).

54. Yuan, J., Ghosal, G. \& Chen, J. The HARP-like domain-containing protein AH2/ZRANB3 binds to PCNA and participates in cellular response to replication stress. Mol. Cell 47, 410-421 (2012).

55. Ait Saada, A. et al. Unprotected replication forks are converted into mitotic sister chromatid bridges. Mol. Cell 66, 398-410.e4 (2017).

56. Berti, M. et al. Human RECQ1 promotes restart of replication forks reversed by DNA topoisomerase I inhibition. Nat. Struct. Mol. Biol. 20, 347-354 (2013).

57. Neelsen, K. J., Zanini, I. M. Y., Herrador, R. \& Lopes, M. Oncogenes induce genotoxic stress by mitotic processing of unusual replication intermediates. J. Cell Biol. 200, 699-708 (2013).

58. Couch, F. B. et al. ATR phosphorylates SMARCAL1 to prevent replication fork collapse. Genes Dev. 27, 1610-1623 (2013).

59. Nakanishi, K., Cavallo, F., Brunet, E. \& Jasin, M. Homologous recombination assay for interstrand cross-link repair. Methods Mol. Biol. 745, 283-291 (2011).

60. Mason, J. M. et al. The RAD51-stimulatory compound RS-1 can exploit the RAD51 overexpression that exists in cancer cells and tumors. Cancer Res. 74, 3546-3555 (2014)

61. Yata, K. et al. Plk1 and CK2 act in concert to regulate Rad51 during DNA double strand break repair. Mol. Cell 45, 371-383 (2012).

62. Mason, J. M. \& Sekiguchi, J. M. Snm1B/Apollo functions in the Fanconi anemia pathway in response to DNA interstrand crosslinks. Hum. Mol. Genet 20, 2549-2559 (2011)

63. Fugger, K. et al. FBH1 co-operates with MUS81 in inducing DNA doublestrand breaks and cell death following replication stress. Nat. Commun. 4 1423 (2013).

\section{Acknowledgements}

This work is funded by the National Institutes of Health grants GM50936 to D.K.B. CA205518-01 to D.K.B, and the Clemson University Open Access Publishing Fund to J.M.M. We thank Alessandro Vindigni for helpful comments on a draft of the paper.

\section{Author contributions}

Y.L.C. purified RAD51 proteins and performed DNA binding assays and D-loop assay J.M.M. performed all cell-based assays. J.M.M., Y.L.C., R.W.W., and D.K.B. contributed to the experimental design and preparation of the paper.

\section{Additional information}

Supplementary Information accompanies this paper at https://doi.org/10.1038/s41467 019-12297-0.

Competing interests: The authors declare no competing interests.

Reprints and permission information is available online at http://npg.nature.com/ reprintsandpermissions/

Peer review information Nature Communications thanks Dana Branzei and other anonymous reviewer(s) for their contribution to the peer review of this work.

Publisher's note Springer Nature remains neutral with regard to jurisdictional claims in published maps and institutional affiliations.

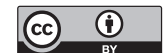

Open Access This article is licensed under a Creative Commons Attribution 4.0 International License, which permits use, sharing, adaptation, distribution and reproduction in any medium or format, as long as you give appropriate credit to the original author(s) and the source, provide a link to the Creative Commons license, and indicate if changes were made. The images or other third party material in this article are included in the article's Creative Commons license, unless indicated otherwise in a credit line to the material. If material is not included in the article's Creative Commons license and your intended use is not permitted by statutory regulation or exceeds the permitted use, you will need to obtain permission directly from the copyright holder. To view a copy of this license, visit http://creativecommons.org/ licenses/by/4.0/.

(C) The Author(s) 2019 